\title{
Disease Surveillance: The Bedrock for Control and Prevention
}

\author{
Bhuvana Krishna
}

Keywords: Coronavirus disease 2019, Infection surveillance, Influenza A (H1N1).

Indian Journal of Critical Care Medicine (2021): 10.5005/jp-journals-10071-23908

"Anything that is measured and watched, improves"-Bob Parsons

The term "surveillance" in French means "sur" (over) and "veiller" (to watch). ${ }^{1}$ In 1963, Alexander Langmuir, the chief epidemiologist of the United States' Centers for Disease Control and Prevention (CDC), defined disease surveillance as "continued watchfulness over the distribution and trends of incidence of diseases by (1) systematic collection of important data, (2) consolidation and interpretation of the data, and (3) dissemination of the results to healthcare professionals and public health policymakers, for appropriate actions." ${ }^{2}$ The earliest surveillance system dates back to Hippocrates, the Father of Medicine and the first epidemiologist, who systematically collected and analyzed data with respect to diseases. $^{3}$

Surveillance objectives and strategies have evolved over the last decade. Early surveillance practices were restricted to "personal surveillance," for enforcement of quarantine in highly contagious diseases, in individuals with history of exposure or who developed early symptoms. ${ }^{4}$

The modern-day practice of surveillance has a broader interest of public health. Public health surveillance is important for the prevention and control of both communicable and noncommunicable diseases. It is a vital tool in the immediate detection of epidemics, in the determination of risk factors and population at risk, and in assessing the burden of the disease and the extent of its spread. It also helps in formulating preventative measures like vaccine development and in framing research priorities. ${ }^{5}$

The World Health Organization (WHO) had established an epidemiological surveillance division in $1965 .^{6}$ This division was responsible for the global coordination of surveillance programs for infectious diseases. The term "surveillance" is often considered synonymous to epidemiology, which includes research and publication. It was Alexander Langmuir who had clearly stated that the primary goal of surveillance was "public health," which includes systematic collection, analysis and dissemination of health data, periodically to all stake holders, with no emphasis on research. ${ }^{7}$ The initial terminology of "epidemiological surveillance" used by WHO was later changed to "public health surveillance" to prevent the dilution of the original benefits of surveillance.

In 2009 during the $\mathrm{H} 1 \mathrm{~N} 1$ influenza pandemic, it was evident that there was very little historic and real-time data regarding patients who were hospitalized for the treatment of severe acute respiratory illness (SARI) or those presenting to the outpatient services with influenza-like illness (ILI). The WHO then established the Global Influenza Program (GIP), which mandated hospital-based SARI and ILI surveillance systems, to define the influenza disease spectrum, population at risk, and disease burden. ${ }^{8}$ The GIP also collates the global surveillance data and provides valuable information on
Department of Critical Care Medicine, St John's Medical College and Hospital, Bengaluru, Karnataka, India

Corresponding Author: Bhuvana Krishna, Department of Critical Care Medicine, St John's Medical College and Hospital, Bengaluru, Karnataka, India, Phone: +91-9945693221, e-mail: bhuvana.11@gmail.com

How to cite this article: Krishna B. Disease Surveillance: The Bedrock for Control and Prevention. Indian J Crit Care Med 2021;25(7):745-746.

Source of support: Nil

Conflict of interest: None

the trends in influenza cases to other parts of the world. Influenza surveillance program is one of the best established till date, by the WHO.

The case definitions of SARI and ILI undergo frequent modifications to improve the sensitivity and specificity of the influenza surveillance program. ${ }^{9}$

The coronavirus disease 2019 (COVID-19) pandemic caused by the severe acute respiratory syndrome coronavirus-2 (SARSCoV-2) have symptoms similar to influenza-fever, cough, and breathing difficulty. Hence, WHO had proposed that the ILI and SARI syndromic surveillance definitions would be sufficient to capture the cases of COVID-19 infections. ${ }^{10}$ Syndromic surveillance in COVID-19 is vital for the early detection and isolation of cases, because of the time lag in availability of laboratory confirmation.

It is also essential to broadcast the analyzed COVID-19 surveillance data so as to estimate the positivity rate and to determine the performance of the influenza syndromic surveillance.

The research paper by Pannu et al. ${ }^{11}$ presents the SARI surveillance data for COVID-19 infection from a tertiary care hospital from North India during the year 2020.

COVID-19 has enlightened the fact that India still lacks robust surveillance programs. The major hurdles faced in India are quality of data, methods of data collection, networking with different regions, and lack of timely analysis and dissemination of the information.

These pitfalls can be overcome by integrating informatics with healthcare systems and with allocation of adequate resources for public health surveillance.

Surveillance programs should be periodically evaluated for its performance. Newer innovations in surveillance methods need to be adopted to develop robust national public health surveillance systems for both communicable and noncommunicable diseases, in India.

(c) Jaypee Brothers Medical Publishers. 2021 Open Access This article is distributed under the terms of the Creative Commons Attribution 4.0 International License (https://creativecommons.org/licenses/by-nc/4.0/), which permits unrestricted use, distribution, and non-commercial reproduction in any medium, provided you give appropriate credit to the original author(s) and the source, provide a link to the Creative Commons license, and indicate if changes were made. The Creative Commons Public Domain Dedication waiver (http://creativecommons.org/publicdomain/zero/1.0/) applies to the data made available in this article, unless otherwise stated. 


\section{OrCID}

Bhuvana Krishna 은 https://orcid.org/0000-0002-0003-6797

\section{References}

1. Choi BC. The past, present, and future of public health surveillance. Scientifica 2012;2012:875253. DOI: 10.6064/2012/875253.

2. Langmuir AD. The surveillance of communicable diseases of national importance. N Engl J Med 1963;268(4):182-192. DOI: 10.1056/ NEJM196301242680405.

3. Eylenbosch WJ, Noah ND, Paterson JG, Stroobant A, Casteren VV, Thiers $\mathrm{G}$, et al. Surveillance in health and disease. Oxford University Press; 1988.

4. Declich S, Carter AO. Public health surveillance: historical origins, methods and evaluation. Bull World Health Organ 1994;72(2):285304. PMID: 8205649; PMCID: PMC2486528.

5. Thacker SB, Stroup DF. Public health surveillance and health services research. In: Epidemiology and health services. Oxford University Press; 1998. p. 61-82.
6. Raskà K, World Health Organization. National and international surveillance of communicable diseases. WHO Chron 1966;20(9):315321. PMID: 5976063. https://apps.who.int/iris/handle/10665/74658

7. Langmuir AD. Communicable disease surveillance: evolution of the concept of surveillance in the United States. J R Soc Med 1971;64(6):681-684. DOI: 10.1177/003591577106400646.

8. World Health Organization. WHO Regional Office for Europe guidance for influenza surveillance in humans. 2009

9. WHO surveillance case definitions for ILI and SARI, as of January 2014. Geneva: World Health Organization; 2020. Available from: www.who. int/influenza/surveillance_monitoring/ili_sari_surveillance_case_ definition/en/.

10. World Health Organization. Public health surveillance for COVID-19: interim guidance. World Health Organization. Available from: https://www.who.int/publications/i/item/who-2019-nCoVsurveillanceguidance-2020.8.

11. Pannu AK, Kumar M, Singh P, Shaji A, Ghosh A, Behera A, et al. Severe Acute Respiratory Infection Surveillance during the Initial Phase of the COVID-19 Outbreak in North India: Comparison of COVID-19 to Other SARI Causes. Indian J Crit Care Med 2021;25(7):761-767. 